\title{
ORDINE E MUTAMENTO NELLE RELAZIONI INTERNAZIONALI
}

\author{
di Alessandro Colombo
}

Se è vero che i silenzi e le omissioni di una disciplina dicono, a volte, più di quanto dicano le sue parole, la riflessione post-bellica delle relazioni internazionali ha meno da dire sul mutamento internazionale di quanto questo abbia da dire sulle relazioni internazionali.

Quando si tireranno le somme della storia della politica internazionale del nostro secolo, infatti, essa apparirà come una successione di mutamenti colossali: dalla fine degli imperi asburgico, ottomano e germanico all'indomani della prima guerra mondiale, a quella degli imperi coloniali dopo la secon$\mathrm{da}$, fino a quella dell'impero russo-sovietico che ha chiuso anche simbolicamente il Novecento. Tanto più sorprendente, quindi, è il fatto che di questi processi non sia rimasta quasi traccia nell'analisi scientifica della politica internazionale. Con alcune lodevoli eccezioni, fino alla fine degli anni settanta la gran parte dell'analisi della politica internazionale si concentrò su elementi statici, quando non finì per essere pura e semplice teoria del bipolarismo. Diversi elementi giocarono a favore di questa scelta (Gilpin 1989, 4-6): la priorità, consueta nelle scienze sociali, dell'analisi statica rispetto all'analisi dinamica (Schumpeter 1979), resa ancora più pervasiva dal successo della teoria dei sistemi; il progressivo declino di quella che K.J. Holsti definì la «grande teoria» (Holsti 1971), cioè dei tentativi di costruire una teoria generale delle relazioni internazionali; la contraddizione tra $\mathrm{i}$ colossali mutamenti che avvenivano nel Terzo Mondo e la matrice euro-occidentale della disciplina; la mancanza, soprattutto, di una «domanda» di teorie del mutamento, annullata anch'essa nel «lungo presente» del confronto bipolare.

Questo tenace silenzio sarebbe già, di per sé, un fatto significativo. $\mathrm{Ma}$ altrettanto se non più significativo è il fatto che,

RIVISTA ITALIANA DI SCIENZA POLITICA / a. XXVII, n. 2, agosto 1997 
per tornare a occuparsi del mutamento, la teoria recente delle Relazioni Internazionali abbia atteso che questo riguardasse (o sembrasse riguardare) gli Stati Uniti e l'Occidente. Per ironia della sorte, la rinascita dell'interesse per il mutamento si deve all'acceso e, visto da oggi, un po' precipitoso dibattito sul «declino americano». L'indebolimento relativo degli Stati Uniti rispetto alle altre potenze, l'esperienza sul campo (in Vietnam, in Iran e in Libano) dell'impossibilità di trasformare il proprio «potere potenziale» in «potere attuale» (Baldwin 1986), la progressiva diminuzione del tasso di polarizzazione del sistema, indussero molti studiosi a ritenere quello di allora (e, per molti versi, anche quello di oggi) un periodo di «erosione dell'egemonia» (Keohane e Nye 1977, 42-46), e a porsi il problema che pochi si erano posti dopo la seconda guerra mondiale: che cosa comporta la crisi di una egemonia? Come avviene il passaggio da una «pace egemonica» a un'altra? Che cosa muove, soprattutto, l'ascesa e il declino delle grandi potenze?

Come spesso accade nello sviluppo delle scienze sociali, nello stesso momento in cui ha sollecitato finalmente una riflessione sul tema del mutamento, la questione del declino americano ha impresso su questa riflessione un ingombrante vizio d'origine. Le teorie che ne sono derivate tradiscono tutte, infatti, lo stesso «punto di vista». Se, in passato, l'analisi scientifica delle Relazioni Internazionali correva il rischio di non trovare posto per la dimensione temporale, il rischio che corre oggi è quello di scambiare una parte per il tutto, condannandosi a non avere nulla da dire su tutto ciò che non rientra in questo orizzonte.

Ordine, egemonia e mutamento internazionale. Le teorie egemoniche

Per i nostri scopi, tre teorie meritano particolare attenzione: la teoria della stabilità egemonica, la teoria dei lunghi cicli, e la teoria della guerra e del mutamento internazionale di Robert Gilpin. Queste teorie, che hanno conquistato (nel bene e nel male) una indubbia centralità nel dibattito recente sul mutamento, si occupano di aspetti diversi della politica internazionale, con una sensibilità assai diversa per la politica, l'economia, e soprattutto la storia. Quello che importa, tuttavia, è che al di là delle differenze esse condividono la medesima struttura, la quale è imperniata su un duplice rapporto: quello tra ordine ed 
egemonia, da un lato, e quello tra crisi dell'ordine egemonico e mutamento internazionale dall'altro. Il primo definisce le condizioni della stabilità, che è associata alla presenza di un paese tanto più forte degli altri da garantire «la pace e la sicurezza internazionale, il godimento dei diritti sul territorio, e la regolazione delle relazioni economiche globali» (Goldstein 1988; Modelski 1987). Il secondo definisce le condizioni del mutamento, ed è solo l'altra faccia del precedente. Se è l'egemonia che produce l'ordine, non può esserci ordine senza egemonia.

a) La teoria della stabilità egemonica, il più delle volte associata ai nomi di Stephen Krasner (1976), Robert Keohane e Joseph Nye (1977) e Charles P. Kindleberger (1973; 1981), costituisce per certi versi la più fortunata tra le analisi dell'egemonia, ma anche quella più distante da una teoria generale del mutamento internazionale. La sua attenzione rimane confinata, infatti, all'economia internazionale, o meglio a un tipo particolare di economia politica internazionale, quella liberal-capitalista, caratterizzata dal libero gioco del mercato nel commercio, nella finanza e negli investimenti. La teoria della stabilità egemonica poggia sull'assunto che esista una stretta relazione tra la struttura del sistema politico globale e il grado di apertura del commercio internazionale. Perché l'economia internazionale sia stabile e aperta, e perché $\mathrm{i}$ «regimi» informali sui quali convergono le aspettative degli attori (Krasner 1986; Young 1991) siano provvisti di un grado adeguato di certezza, è necessario che vi sia uno Stato più forte degli altri e che esso continui a fornire disponibilità finanziarie, a favorire l'instaurarsi di un sistema di tassi di cambio relativamente stabili, a garantire il coordinamento delle politiche macroeconomiche, ad agire, al manifestarsi di crisi finanziarie, come prestatore di ultima istanza. A mano a mano che la potenza egemone si indebolisce rispetto agli altri stati e perde la capacità e/o la volontà di svolgere il proprio ruolo, invece, il libero scambio dei beni, dei capitali e dei servizi lascia posto a politiche protezionistiche, e nella peggiore delle ipotesi alla totale chiusura dei mercati. La diffusione di potenza è associata all'incertezza e al conflitto. Come afferma Kindleberger $(1973,305)$, «perché l'economia internazionale sia resa stabile è necessario qualcuno che la stabilizzi, una potenza stabilizzatrice».

Dietro l'etichetta di «teoria della stabilità egemonica», coniata inizialmente da Robert Keohane (1980), è raggruppato un gruppo assai eterogeneo di studiosi. Alcuni elementi possono 
essere considerati, tuttavia, rappresentativi del loro concetto di egemonia. In primo luogo, l'accento sulla superiorità economica, finanziaria e commerciale, piuttosto che su quella politicomilitare ${ }^{1}$. In secondo luogo, il sottinteso liberale. La semplice esistenza di una potenza egemonica non è ritenuta sufficiente ad assicurare lo sviluppo di un'economia internazionale aperta. Perché ciò avvenga, è necessario che la potenza egemone si ispiri ai valori del liberalismo; che la stessa congruenza di scopi sociali in supporto a un sistema liberale sia presente tra le maggiori potenze economiche; che lo stesso interesse allo sviluppo di relazioni di mercato sia condiviso dalle altre potenze. Infine, la teoria della stabilità egemonica tende a vedere nell'egemonia un'espressione di benevolenza più che di dominio. Sebbene l'egemone tragga i propri vantaggi dall'esercizio del suo ruolo, infatti, gli stati minori guadagnerebbero proporzionalmente ancora di più, non sopportando che in piccola parte i costi di fornitura dei beni pubblici, ma godendone tutti i benefici.

b) La teoria dei lunghi cicli costituisce il tentativo più ambizioso di costruire una teoria comprensiva dell'ordine, dell'egemonia e del mutamento internazionale. Malgrado le perplessità che è possibile esprimere sull'uso disinvolto dei dati empirici, e sulla pretesa di inferire da semplici generalizzazioni storiche nientedimeno che il «ritmo della politica globale» (Modelski $1987,1)$, ci sono almeno due ragioni per considerarla come l'esempio più rappresentativo dell'analisi recente del mutamento internazionale.

La prima ragione è che, a differenza della teoria precedente, questa concettualizza esplicitamente il tema del mutamento in termini di concentrazione e deconcentrazione della potenza, e cioè negli stessi termini della maggior parte dello studio postbellico delle Relazioni Internazionali. L'esistenza dei «lunghi cicli dell'egemonia» non è considerata alla stregua di un semplice fatto empirico, ma è spiegata volta per volta o come l'effetto di una tendenza all'entropia presente in qualunque tipo di ordine (Kumon 1987, 60), oppure sulla base del rapporto tra la do-

1 «Per essere considerato egemone nell'economia mondiale» scrive Robert Keohane $(1984,33-34)$ «un paese deve avere accesso a materie prime cruciali, deve detenere il controllo sulle maggiori fonti di capitale, deve mantenere un grande mercato domestico per le importazioni e, infine, deve possedere un vantaggio comparato nella produzione di beni ad alto valore tecnologico e strategico che garantiscono alti livelli di salari e di profitti. Esso deve anche essere superiore ad ogni altro paese, rispetto a tutte queste dimensioni considerate nel loro insieme». 
manda e l'offerta complessiva di ordine internazionale (Thompson e Modelski 1987), oppure più frequentemente come l'erosione progressiva di una posizione di monopolio. La tesi di Modelski e Thompson è che ogni «lungo ciclo» della politica internazionale sia segnato dallo spostamento, lungo la dimensione della polarità, da una posizione di bassa concentrazione (che corrisponde, come vedremo, alla guerra) a una di alta concentrazione (l'egemonia mondiale) e infine, nuovamente, a una posizione di bassa concentrazione (Modelski 1987, 5). La distribuzione delle potenzialità tra gli attori è decisiva tanto per l'egemonia quanto per l'ordine internazionale. Quando la potenza è più concentrata - all'indomani della «guerra generale» - l'egemone non ha difficoltà a fornire il «bene pubblico» della sicurezza e a garantire le relazioni economiche internazionali. A mano a mano che la politica internazionale si sposta verso la deconcentrazione, invece, il paese più forte diventa meno capace di svolgere il proprio ruolo, mentre uno o più attori possono riuscire a dimostrare di essere in grado di non obbedirgli senza che la disobbedienza venga sanzionata (Bonanate 1986, 127).

La seconda ragione, che si impone da sé, riguarda l'immagine del tempo. Indagando la dimensione temporale dei processi politici internazionali (chronomacropolitics), la teoria dei lunghi cicli offre una ricostruzione di lungo periodo della storia delle relazioni internazionali, rigettando in modo esplicito le visioni lineari della storia ${ }^{2}$. La tesi sulla quale poggia è che la politica internazionale non proceda né verso la concentrazione né verso la deconcentrazione, ma segua cicli regolari di mutamento, nel corso dei quali l'ordine internazionale governato dalla potenza egemone si incrina progressivamente fino ad essere sostituito da un nuovo ordine internazionale governato da una nuova potenza egemone.

Questi «lunghi cicli» sono scansioni ripetitive della politica internazionale, di durata variabile tra cento e centocinquant'anni, e divisi a propria volta in quattro fasi di venticinquetrent'anni.

La prima, di bassa concentrazione e di estremo disordine, consiste nella lotta tra le principali potenze per assumere il go-

2 Tentativi di individuare cicli regolari di mutamento internazionale, con particolare riferimento alla guerra, non mancano neppure nella teoria precedente. Per alcuni esempi di riflessione su questo tema, cfr. Toynbee (1934), Wright (1965), Sorokin (1937). 
verno del sistema (global war). La «guerra globale» rappresenta la cerniera tra vecchio e nuovo ordine, e l'evento costitutivo di ciascun ciclo. A differenza delle guerre ordinarie, infatti, essa non sposta gli equilibri all'interno di un dato sistema internazionale, ma determina «chi organizzerà il nuovo ordine e secondo quali principi».

La seconda fase (world power) è quella nella quale si sperimenta il massimo della concentrazione di potenza e della legittimità del nuovo ordine. I trattati di pace che lo formalizzano (come quelli di Vienna del 1815, o le Conferenze inter-alleate del 1943-45), e che stanno al sistema internazionale come la Costituzione sta al sistema interno, rappresentano infatti il momento nel quale è maggiore sia la domanda di ordine e di stabilità da parte dei membri della convivenza internazionale, sia la capacità del più forte di offrire quello che gli altri gli chiedono.

La terza fase (delegitimation) inaugura il processo degenerativo dell'egemonia. A mano a mano che si affievolisce la memoria della guerra e della ricostruzione, l'ordine internazionale e il suo custode patiscono una crescente crisi di legittimità. Da un lato, la domanda di ordine deve fare i conti con altre domande, spesso di segno contrario, provenienti dalle potenze in ascesa nel nuovo sistema internazionale. Dall'altro lato, i costi del ruolo globale diminuiscono a poco a poco anche l'offerta di ordine del più forte.

Nella quarta fase (deconcentration), infine, la crisi dell'egemonia si acuisce, e le richieste di rinegoziazione dell'ordine diventano più intense e di portata sempre più ampia (Bonanate 1986, 126-127). La redistribuzione delle potenzialità tra gli attori trasforma la crisi della legittimità in aperta contestazione. Nello stesso momento in cui erode la supremazia dell'egemone, la deconcentrazione provoca l'emergere di uno sfidante (o di un gruppo di sfidanti) che aspira a prendere il suo posto. Il conflitto acquista intensità, fino a sfociare in un'altra guerra generale che chiude il lungo ciclo e ne apre uno nuovo.

c) La teoria della guerra e del mutamento internazionale di Robert Gilpin sembra volere tenere conto di uno spettro più ampio di trasformazioni. Al principio della sua analisi, infatti, Gilpin distingue tre tipi diversi di mutamento internazionale. Il primo e fondamentale è il mutamento nella natura degli attori che compongono il sistema internazionale, come quello che ebbe luogo con l'ascesa e il declino delle città-Stato greche ( $s y$ stems change). Il secondo tipo, che coincide grosso modo con 
quello analizzato da Organski, è il mutamento nella forma di controllo o di governo del sistema internazionale (systemic change). Il terzo tipo, infine, è costituito da tutti i mutamenti che avvengono nelle interazioni tra gli attori (politica delle alleanze, relazioni economiche, sistemazioni territoriali) all'interno di un dato sistema internazionale (interaction change).

Malgrado queste premesse, tuttavia, anche Robert Gilpin finisce per occuparsi del solo tipo di-mutamento che interessasse l'establishment scientifico e l'opinione pubblica americani tra la fine degli anni settanta e l'inizio degli anni ottanta: il declino delle potenze egemoni. «La storia di un sistema internazionale» scrive Gilpin $(1989,86)$ «è la storia dell'ascesa e del declino degli imperi e degli stati che, durante il loro periodo di dominio degli affari internazionali, hanno dato ordine e stabilità al sistema... L'essenza del mutamento sistemico consiste dunque nella sostituzione di una potenza dominante in declino con una in ascesa».

Il motore del processo è la contraddizione tra le componenti essenziali dell'ordine internazionale vigente e la capacità della potenza egemone di mantenerlo, tra i costi per la difesa della distribuzione del territorio, delle regole e dell'economia internazionale, da un lato, e le entrate necessarie per finanziarla dall'altro (Gilpin 1989, 224). Nel momento in cui una potenza impone la propria egemonia, il governo del sistema riflette in pieno la gerarchia tra gli attori, e la potenza egemone ha la capacità e l'interesse di strutturare il sistema internazionale secondo i propri interessi. Da questo momento, tuttavia, i costi per il mantenimento dello status quo tendono a crescere più rapidamente della capacità di finanziarlo. Questo divario tra costi e risorse provoca una «crisi fiscale» della potenza egemone, e infine uno squilibrio tra la distribuzione delle potenzialità tra gli attori, che è la più soggetta al mutamento, e la distribuzione dei benefici politici, economici e culturali, che continua a riflettere il predominio della potenza in declino.

Se questa situazione di squilibrio non si risolve, l'ordine internazionale entra in crisi. Come già per Organski ${ }^{3}$, anche per

3 Anche per la teoria del trasferimento di potenza di Organski, la concentrazione del potere militare nelle mani di un solo attore ha effetti stabilizzanti sul sistema internazionale, mentre l'instabilità deriva dal progressivo indebolimento del più forte e dalla redistribuzione del potere tra più attori. Una guerra generale diventa più probabile quando il potere militare delle potenze emergenti comincia ad avvicinarsi a quello della 
Gilpin lo strumento proprio della politica internazionale per risolvere lo squilibrio tra la struttura del sistema e la redistribuzione del potere è la guerra per l'egemonia, che è l'equivalente della rivoluzione nella politica interna ${ }^{4}$. Ad essa spetta il compito di creare «un nuovo ordinamento delle componenti basilari del sistema. La vittoria e la sconfitta ristabiliscono senza ambiguità la gerarchia di prestigio consona alla nuova distribuzione di potere nel sistema. La guerra stabilisce chi governerà il sistema internazionale e quali interessi saranno avvantaggiati dal nuovo ordine internazionale. La guerra procura inoltre una ridistribuzione del territorio tra gli stati del sistema, fissa una nuova serie di regole, muta la divisione internazionale del lavoro» (Gilpin 1989, 273-274).

\section{Equilibrio ed egemonia}

Quando, nella riflessione del passato, la politica internazionale veniva designata come un ambiente anarchico, cioè privo di governo, per assenza di governo non si intendeva affatto che la politica internazionale si svolgesse all'ombra del disordine (Miller 1992), ma solo che l'ordine poteva essere, e storicamente era, ottenuto in modi diversi da quelli propri della politica interna. In una celebre tripartizione del fenomeno-pace, Raymond Aron propose di distinguere le paci storiche sulla base di tre diversi rapporti di forza:

potenza egemone: «Le nazioni evitano di combattere a meno che esse non ritengano di avere buone probabilità di vittoria, ma questo è vero per entrambe le parti solo quando le loro capacità sono grosso modo uguali o almeno sono percepite come tali» (Organski 1968 , 294). La maggiore probabilità della guerra, e l'instabilità del sistema che ad essa viene associata, derivano dalla difficoltà di calcolare in anticipo modalità ed esito del conflitto. Ogni volta che si verifica un «trasferimento di potenza» tra due o più attori, «sia la potenza emergente sia quella egemone possono avere difficoltà a misurare la propria potenza relativa e possono restare coinvolti in una guerra che non avrebbe mai avuto luogo se entrambe le parti avessero avuto modo di fare previsioni accurate» (Organski 1968, 480).

4 L'eventualità più probabile è che sia lo sfidante ad aprire le ostilità per accelerare la transizione e assicurarsi benefici e influenza più adeguati al suo peso. Ma non è escluso, come dimostra l'esperienza storica, che sia l'egemone in declino a scatenare una guerra preventiva prima che lo sfidante possa eguagliarlo o superarlo. L'esempio classico di guerra preventiva rimane, naturalmente, la Guerra del Peloponneso, secondo la nota interpretazione di Tucidide $(1,23)$ : «Il motivo più vero, ma meno dichiarato apertamente, penso che fosse il crescere della potenza ateniese e il suo incutere timore ai Lacedemoni, sì da provocare la guerra» (Tucidide 1989, 111). 
Poiché i rapporti di potenza in tempo di pace, pur senza essere il riflesso esatto del rapporto di forze attuali o potenziali, sono un'espressione più o meno deformata di esso, i diversi tipi di pace possono essere riferiti ai tipi di rapporti di forze. Distinguo tre tipi di pace, equilibrio, egemonia, impero: in un dato spazio storico, le forze delle unità politiche o si controbilanciano o sono dominate da quelle di una di esse, oppure infine sono superate da quelle di una di esse in modo che tutte le unità, salvo una, perdono la loro autonomia e tendono a sparire in quanto centri di decisioni politiche $(1970,188)$.

Nelle teorie egemoniche del mutamento, al contrario, l'ordine internazionale appare inconcepibile senza che qualcuno si assuma ciò che di volta in volta è definita «funzione direttiva» (Tucker 1981, 15), «potere strutturale» (structural power) (Christensen 1977), «meta-potere» (Baumgartner et al. 1975), o puramente e semplicemente «governo del sistema» (Gilpin 1989)5. Delle diverse forme di ordine internazionale del passato, le teorie dell'egemonia ne salvano soltanto una. Mentre, nella riflessione tradizionale, l'ordine egemonico era solo una delle modalità dell'ordine internazionale, nelle teorie più recenti l'ordine internazionale o è egemonico o non è.

Questo spostamento d'accento spiega perché, e in che cosa, le teorie egemoniche si discostino dalle teorie tradizionali del mutamento internazionale, quelle dell'equilibrio. A prima vista, i rispettivi punti di partenza non sono troppo distanti. Tanto le une quanto le altre sono varianti della teoria realista della politica internazionale (Levy 1990), della quale condividono l'interesse per il potere e per il modo in cui è distribuito tra gli attori. Senonché, invece di indulgere nell'interminabile discussione sulla maggiore o minore stabilità e/o war proneness dei sistemi politici internazionali, lo studio del mutamento internazionale si concentra sulla dinamica dei rapporti di potere nel tempo piuttosto che sulla distribuzione statica del potere all'interno del si-

5 Questo dominio sulle regole riguarda tanto la dimensione economica quanto la dimensione politica quanto quella culturale o ideologica. Non è facile stabilire a quale di queste dimensioni spetti la priorità. Alcuni autori, come Wallerstein (1982) e Braudel (1966), danno più peso alla superiorità economica, e suggeriscono una immagine per sfere concentriche dell'economia internazionale, al centro della quale sorge lo Stato o la città dominante. Altri studiosi, di marca più o meno realista (Organski 1968; Gilpin 1989; Modelski 1978), preferiscono concentrare la propria attenzione sulla superiorità militare, e vedono nell'egemone la potenza che sta «al vertice della piramide» (Organski e Kugler 1980, 19), in una posizione diversa rispetto alle altre grandi potenze che hanno meno capacità di influenzare i paesi minori e ricevono meno benefici dall'ordine internazionale. Comunque sia, egemonia economica ed egemonia politico-militare hanno bisogno l'una dell'altra: la prima serve a finanziare la seconda, e la seconda a vigilare sulla prima. 
stema (bipolare o multipolare) (Gilpin 1989, 147-148). È la crescita differenziata o diseguale del potere tra gli stati a pregiudicare la stabilità dei sistemi internazionali, mettendo in contraddizione la distribuzione dei benefici con quella della potenza.

Da questa base comune, tuttavia, le teorie dell'equilibrio e quelle dell'egemonia ricavano una diversa immagine del sistema internazionale, una diversa descrizione della sua storia, e una diversa spiegazione dei fattori del mutamento. Il primo motivo di disaccordo affonda le proprie radici nella diversa definizione della struttura del sistema internazionale: anarchia nel primo caso, gerarchia nel secondo (Bonanate 1986). Nelle teorie dell'equilibrio, nelle quali il sistema internazionale è considerato composto di stati separati, autonomi e, soprattutto, formalmente eguali, l'insistenza sulla struttura orizzontale della politica internazionale relega fatalmente ai margini il concetto di egemonia mondiale, che presuppone una struttura verticale di carattere gerarchico. Nella visione tradizionale della politica internazionale, anche quando viene presa in considerazione, l'egemonia è vista come un fatto patologico, anzi proprio come il fatto patologico che il funzionamento «normale» dell'equilibrio si propone di scongiurare (Dehio 1988). Sia che venga interpretato come una situazione nella quale gli stati si trovano a causa della natura anarchica del sistema internazionale, o come una politica che gli stati adottano o dovrebbero consapevolmente adottare (Cesa 1990), l'equilibrio esclude, o combatte, l'instaurazione di qualunque egemonia.

Le teorie più recenti del mutamento internazionale, al contrario, elevano l'egemonia a concetto centrale delle relazioni internazionali (Rapkin 1987, 129). Invece di porre l'accento sulla formale eguaglianza degli stati, liquidando le relazioni ineguali tra stati dominanti e subordinati come relazioni a loro volta «anarchiche», le teorie dell'egemonia concentrano la propria attenzione sulle strutture di autorità presenti nel sistema internazionale, interpretandole non solo come relazioni materiali ma anche, e soprattutto, come relazioni sociali (Wendt e Friedheim 1995, 690). Dalla loro prospettiva, la storia del sistema mondiale - quello nato a cavallo del Cinquecento con l'espansione europea e la lenta formazione di un unico «sistema capitalistico mondiale» (Wallerstein 1982) - non può essere compresa nei consueti termini orizzontali, senza fare riferimento a uno Stato separato dagli altri sia per $\mathrm{i}$ fini che si propone sia per i mezzi che impiega (Rapkin 1987; Kumon 1987). Condizioni e natura 
del mutamento internazionale risultano specularmente opposti rispetto alle teorie dell'equilibrio. In un caso, l'equilibrio è la condizione «normale» e l'egemonia il fatto patologico; nell'altro caso, invece, è l'equilibrio ad essere storicamente eccezionale, mentre di norma c'è sempre una potenza che domina il sistema internazionale. La struttura del sistema, che nelle teorie dell'equilibrio cambia ogni volta che cambia il numero dei poli, in queste teorie si può dire che non cambi mai. L'unica cosa che cambia è la potenza al vertice della piramide ${ }^{6}$.

Da queste diverse immagini discendono due modi altrettanto diversi di definire $\mathrm{i}$ fattori del mutamento internazionale. Nelle teorie dell'equilibrio l'eguaglianza delle forze (tra le grandi potenze) è associata alla stabilità, mentre il motore del mutamento è l'eccessivo rafforzamento di uno Stato rispetto agli altri. Il sistema politico internazionale si muove secondo l'alternanza equilibrio-crisi-nuovo equilibrio: ogni volta che uno Stato diventa così forte da minacciare di ottenere una posizione di predominio sull'intero sistema internazionale, le altre grandi potenze sono indotte a coalizzarsi per prevenirne l'espansione e ristabilire l'equilibrio (Toynbee 1934; Dehio 1988).

Nelle teorie dell'egemonia, al contrario, il rischio dell'instabilità e della guerra non deriva dallo strapotere del più forte, ma dal riequilibrio con i più deboli. Il modello teorico dell'egemonia si realizza in termini di tensione tra ordine, crisi e nuovo ordine (Bonanate 1986, 126-127). L'idea centrale, che discende da una osservazione empirica, è che l'esito delle guerre (costituenti) determini contemporaneamente quale Stato occuperà «la posizione di legislatore della sistemazione post-bellica» (Modelski 1978, 217), e quale contenuto di ordine (politico, econo-

6 Le teorie egemoniche non hanno, tuttavia, lo stesso orizzonte temporale (Rapkin 1987, 139): Gilpin e la teoria della stabilità egemonica (Kindleberger 1973; 1981; Hirsch e Doyle 1977) considerano soltanto l'egemonia americana e quella inglese, Wallerstein (1982) include anche l'Olanda del sedicesimo secolo, Modelski e Thompson si spingono indietro fino alla controversa egemonia portoghese. Tanto l'elenco delle potenze egemoni quanto quello delle guerre generali rimangono molto controversi. Molti studiosi contestano, per esempio, l'esistenza di una egemonia portoghese, o perché ritengono che altri paesi fossero più forti del Portogallo (come Wallerstein, che insiste sulla superiorità navale della Spagna), o perché preferiscono sottolineare il ruolo che singole città, piuttosto che interi stati, svolgevano nell'economia del tempo (Braudel 1966), oppure perché ritengono che proprio questo disaccordo tra gli studiosi sia la prova che nessuna potenza fosse effettivamente egemone (Goldstein 1988, 296). Altri studiosi non concordano sul numero delle guerre generali. Per una breve rassegna delle diverse teorie, cfr. Levy (1986). 
mico e ideologico) detterà agli altri stati. Di qui la dinamica caratteristica del sistema politico internazionale, il quale è destinato a una sorta di degenerazione continua: l'ordine iniziale è anche il massimo possibile, mentre da questo momento «l'ordine dato tende a ridursi, producendo richieste di rinegoziazione di sempre più ampia portata, fino al limite di rottura dell'incompatibilità tra un'ulteriore richiesta e il margine di ordine ancora resistente» (Bonanate 1986, 127).

La guerra gioca, in entrambe le impostazioni, un ruolo fondamentale. Senonché, mentre nelle teorie tradizionali essa è il mezzo per ristabilire la condizione di partenza, l'equilibrio, prevenendo il rischio dell'egemonia, nelle teorie più recenti la guerra è il meccanismo proprio della politica internazionale per selezionare «chi dovrà organizzare il mondo e in che modo». Per riassumere la stessa cosa in forma apodittica: la guerra, che nelle teorie dell'equilibrio ha un ruolo conservatore, nelle teorie dell'egemonia acquista un ruolo costituente.

Questo spostamento della prospettiva comporta due modi altrettanto diversi di leggere il passato. Nella storia degli ultimi quattrocento anni, Modelski e Thompson individuano cinque guerre generali, ciascuna della quali è fonte del «lungo ciclo» di una potenza egemone: la prima parte delle guerre italiane (1494-1517), da cui scaturì l'egemonia portoghese; la guerra di indipendenza olandese (1585-1609), che conferì alle Province Unite l'egemonia mondiale; le guerre di Luigi XIV (1689-1715), che trasferirono l'egemonia all'Inghilterra; le guerre della Rivoluzione francese e quelle napoleoniche (1792-1815), che inaugurarono un nuovo ciclo egemonico inglese; le due guerre mondiali del Novecento (1914-1945), dalle quali sono emersi come nuova potenza egemone gli Stati Uniti.

Questa suddivisione della storia europea in cicli, contrassegnati dal tentativo di una potenza di riunificare il continente europeo e di lanciare da qui la sfida alla potenza egemone su scala mondiale, coincide grosso modo con quella continentalerealistica suggerita da storici quali Arnold Toynbee (1934) e Ludwig Dehio (1988). Tanto più significativo, quindi, è il fatto che non rimangano le stesse le potenze al centro della scena: le potenze vincitrici in un caso, quelle aspiranti all'egemonia continentale, ma uscite sconfitte nelle grandi guerre egemoniche, nell'altro.

La successione delle potenze egemoniche e dei loro sfidanti delinea, dalle due prospettive, due storie diverse. Quella conti- 
nentale-realistica interpreta la storia degli ultimi tre secoli come una successione di tentativi egemonici falliti: quello della Spagna asburgica, quello della Francia di Luigi XIV, quello della Francia napoleonica e quello della Germania guglielmina prima, e hitleriana poi. Quella più recente, al contrario, la interpreta come un continuo passaggio delle consegne tra i vincitori l'Olanda, la Gran Bretagna per due volte, gli Stati Uniti - o per meglio dire tra coloro che beneficiarono delle rispettive sfide ${ }^{7}$.

\section{Una certa idea dell'ordine. L'orizzonte globale della world power}

Ma sapere che le teorie egemoniche del mutamento internazionale si discostano da quelle tradizionali dell'equilibrio non coglie ancora la cosa fondamentale. Quello che conta di più, e rivela meglio di tutto l'orizzonte nel quale si muovono, è il fatto che la loro idea dell'egemonia e dell'ordine internazionale sottintende una visione dello spazio radicalmente diversa da quella della tradizione continentale europea e da quella che domina altri settori delle Relazioni Internazionali.

Il concetto e la pratica dell'egemonia sono tutt'altro che un portato della riflessione più recente. $\mathrm{Al}$ contrario, la stessa parola «egemonia» - calco latino del verbo greco egemonein, che significava letteralmente «andare innanzi», «precedere», «guidare» - racchiude dentro di sé un'origine remota, nella quale sono già riconoscibili due dei suoi tratti fondamentali: il sottinteso militare, simboleggiato nel significato che il termine acquistò nel linguaggio diplomatico greco, nel quale indicava il «diritto di comandare in campo» (più precisamente, di «condurre in campagna») un esercito di coalizione (Miglio 1988, 1035); e la diversità rispetto ad altre forme di diseguaglianza, in modo particolare rispetto all'impero (Doyle 1986, 54-81). Nell'accezione tradizionale, l'egemonia è il potere di fatto che lo Stato

7 Anche quando comportarono un trasferimento dell'egemonia, infatti, le guerre generali degli ultimi quattro secoli si conclusero con un risultato paradossale: la nuova potenza egemone non fu mai lo sfidante della potenza in declino, ma un alleato di quest'ultima (Modelski 1987; Goldstein 1988). Così, come osserva Modelski (1978), prima di diventare il centro dell'egemonia olandese Anversa fu il cuore bancario e commerciale del sistema portoghese. Successivamente, l'Olanda riuscì a resistere alla sfida lanciatale, fino al 1713, dalla Francia di Luigi XIV, ma a prezzo di cedere l'egemonia agli alleati inglesi. La stessa sorte è toccata, infine, alla Gran Bretagna, costretta a trasferire l'egemonia al suo alleato più forte all'indomani della seconda guerra mondiale. 
più forte esercita sugli stati più deboli, ispirandone o condizionandone le scelte sino al punto da costituire un modello per le comunità egemonizzate (Belligni 1983). Come tale, essa non è né un sistema accentrato di tipo imperiale né un sistema rigidamente decentrato secondo i canoni dell'anarchia internazionale (Kumon 1987, 59). A differenza dell'equilibrio, l'egemonia è una situazione nella quale «le forze delle unità politiche sono dominate da quelle di una di esse» (Aron 1970, 188). A differenza dell'impero, invece, questo dominio si esercita fuori del diritto, come pura situazione di fatto, cioè non richiede il controllo diretto dello spazio pacificato (Wallerstein 1982; Modelski 1978, 222).

Ciò può essere espresso in due modi diversi e complementari. $\mathrm{Da}$ un lato, questo significa che la sovranità formale degli stati più deboli non è negata, anche se può andare soggetta a diverse forme di limitazione (Miglio 1988). Come riassume molto efficacemente Julien Freund (1986, 129), a differenza dell'impero, il quale sopprime le sovranità minori a beneficio di quella dominante, l'egemonia rispetta almeno formalmente le sovranità e non manifesta direttamente la propria presenza se non nel caso d'eccezione.

Dall'altro lato, questo significa che l'ordine egemonico ha una propria estensione spaziale, ma è privo di una vera e propria base territoriale. La diminuzione di importanza del controllo e dell'espansione territoriale è, anzi, un Leit-motiv della riflessione sull'egemonia. L'Olanda del XVII secolo, la Gran Bretagna del XVIII e del XIX e gli Stati Uniti - che sono, per la maggior parte degli studiosi, le potenze che si sono succedute nel ruolo di egemoni - non hanno mai cercato di creare un impero universale, anzi la loro politica (di «riequilibratori») ha sempre avuto come obiettivo quello di preservare il sistema degli stati (Chase-Dunn 1990, 120).

L'assenza dell'appropriazione territoriale (Landsnabme) è il primo e fondamentale filo che lega l'egemonia allo spazio. L'egemonia non colloca recinzioni, pietre di confine o mura. Essa domina su uno spazio, ma senza appropriarsene nel senso politico-giuridico del termine. La sua immagine superficiale, e uno dei motivi dominanti della polemica ideologica anti-egemonica, è l'invisibilità. L'egemonia, a differenza dell'espansione territoriale, «non appare». Può rafforzarsi, o conquistare nuovi spazi, ma, qualunque cosa ottenga, non ha mai lo stesso grado di «evidenza» di una appropriazione territoriale. «L'egemonia 
marittima» - come scrive Ludwig Dehio (1988, 92, 122 e 193) guardando dal centro del continente - «si può estendere in modo meno appariscente dell'egemonia terrestre»; «l'egemonia marittima non ha la stessa capacità di spaventare che ha l'egemonia terrestre»; «alla supremazia marittima è permesso di essere più generosa di quel che deve essere la supremazia terrestre!» La polemica anti-inglese degli scrittori francesi dell'epoca delle guerre napoleoniche e di quelli tedeschi di cento anni più tardi ruota in maniera insistente attorno a questo argomento. La mancanza dell'appropriazione territoriale cambia di segno a seconda che venga guardata dall'isola o dal continente. In un caso, essa appare come l'espressione di una superiorità morale, o quanto meno di un grado più elevato di sviluppo politico. Nell'altro caso, invece, essa viene paventata come un formidabile travestimento del dominio:

Il libero cittadino del prospero Stato olandese, il ricco signore del mare, guarda con compassionevole disprezzo gli stati autoritari del continente, i loro tiranni, la loro costrizione delle coscienze, il loro militarismo. Egli si crede in possesso d'una morale politica superiore, come patrocinatore di un ordine mondiale, razionale-illuminato, di pacifica giustizia contro la brutale violenza di guerre assurde - di guerre continentali cioè; guerre marittime invero, condotte per la libertà dei mari, vale a dire per spezzare a vantaggio dell'Olanda il monopolio spagnolo in oltremare, quelle sono un altro conto (Dehio 1988, 71-72).

A questo primo aspetto spaziale le teorie recenti del mutamento internazionale ne aggiungono altri, che costituiscono altrettanti elementi distintivi tra la loro nozione di egemonia e quella tradizionale.

Il primo, e il più sorprendente, riguarda le dimensioni. Nell'accezione tradizionale, così come nella nostra comune esperienza storica, l'egemonia è il predominio che qualunque tipo di Stato può acquistare sugli altri stati all'interno di qualunque spazio storico. Un classico caso di egemonia regionale, per esempio, è quello citato da Aron del continente americano, nel quale la pace «è il risultato duraturo della sproporzione, dovuta alla situazione geografica e accentuata dalla storia, tra le forze» (Aron 1970, 189) degli Stati Uniti e quelle degli altri. Ma esempi analoghi si possono trovare nella storia di tutte le aree regionali, nelle quali non di rado non fu l'equilibrio, ma l'egemonia di un attore nettamente più forte a «tenere in ordine» attori medi e piccoli: basti pensare al ruolo della Germania bismarckiana nel centro del continente europeo, a quello dell'Impero 
cinese nello spazio circostante i propri confini oppure, per tornare all'origine del concetto, all'egemonia di Sparta nella penisola del Peloponneso e a quella di Tebe nella Grecia centrale.

La stessa immagine di un mondo composto da numerosi spazi, in ciascuno dei quali gli attori gravitano attorno all'attore principale senza perdere (almeno in parte) la propria indipendenza, ricorre in varie forme nella storia e nell'analisi più recente della politica internazionale ${ }^{8}$.

Una variante classica è quella delle sfere di influenza, che alcuni autori arrivano a concepire come strutture quasi feudali (Galtung 1966), poste sotto il dominio di una grande potenza la quale mantiene l'ordine tra le potenze minori all'interno della propria sfera e limita, nello stesso tempo, la penetrazione delle altre potenze. A propria volta, questa struttura feudale può essere raffigurata in forma piramidale, collocando $\mathrm{i}$ «pezzi grossi» (top dogs) al vertice a interagire prevalentemente fra loro e con le piccole potenze che si trovano all'interno della propria sfera (Russett e Starr 1992, 129-130), oppure secondo un «modello stellare» (Santoro 1995) che vede la potenza egemone al centro della propria costellazione, e che ha il vantaggio di suggerire meglio il carattere «aperto» dell'egemonia e la mancanza di demarcazioni nette tra una costellazione e l'altra.

Un'altra variante, che accomuna tutte le potenze egemoni indipendentemente dalla loro conformazione interna e dalla loro presunta «vocazione geopolitica», è la pretesa di possedere un ambito di sovranità spaziale che travalica i confini dello Stato, e che designa il limite dei propri interessi prioritari (Schmitt 1991, 369). La designazione dello spazio più prossimo ai propri confini - l'«estero vicino» del Caucaso, dell'Asia Centrale e dell'Europa Orientale per la Russia, il backyard dell'America Centrale e Meridionale per gli Stati Uniti, quello dell'Europa Centrale per la geopolitica tedesca - come di un luogo che non gode di piena autonomia, da un lato, e nel quale si sperimenta il massimo della vulnerabilità, dall'altro, edifica uno spazio di

8 Una variante scopertamente polemica nei confronti dei concetti globali di ordine è quella proposta dalla geopolitica tedesca a partire dalla fine dell'Ottocento (Portinaro $1982 \mathrm{~b}, 21)$. Nell'idea di «grandi spazi organizzati» che avrebbero dovuto succedere alla convivenza ottocentesca tra stati confluivano l'aspirazione all'autarchia legata all'esperienza della precaria posizione geografica della Germania; la diffidenza, o l'ostilità, nei confronti della globalizzazione dell'economia e del pensiero politico-giuridico universalistico; infine, la polemica contro il latore dell'«ordine mondiale», polemica che fa dell'imperialismo il problema di fondo della geopolitica (Grabowsky 1960). 
passaggio tra interiorità ed esteriorità. $\mathrm{Da}$ questo angolo di visuale, il «Lebensraum» della geopolitica tedesca appare come l'equivalente funzionale dell'«estero vicino» russo e dell'«Emisfero occidentale» americano'.

A queste immagini parziali (e plurali), il nuovo concetto di egemonia oppone un'immagine rigidamente unitaria dell'ordine internazionale. Diversamente dall'accezione tradizionale, in questa nuova accezione egemone è soltanto chi esercita il proprio predominio sull'intero sistema internazionale. L'egemonia è sempre, per definizione, egemonia mondiale (world power), per la stessa ragione per la quale è la globalità che determina l'orizzonte dei suoi tre elementi costitutivi: la superiorità economica, che è legata meno alle dimensioni, o all'innovazione per il gusto dell'innovazione, che all'innovazione diretta a una proiezione globale e al commercio mondiale; la superiorità ideologica, cioè il possesso di un linguaggio «universale» capace di «indurre le potenze minori a partecipare alla sua visione dell'ordine internazionale e ad abbracciare come propri... i principi e le norme enunciati dall'egemone» (Ikenberry e Kupchan 1990, 52); la superiorità politico-militare, infine, che è ciò che consente all'egemone di spingersi ovunque si sia già spinto il commercio ${ }^{10}$, differenziandolo alla radice dai suoi sfidanti regionali. Le «grandi potenze» continentali possono anche disporre di capacità (economiche e militari) straordinarie, ma se vogliono aspirare all'egemonia devono procurarsi una organizzazione politico-strategica capace di globalità.

Il secondo tratto distintivo, e la seconda caratteristica dei nuovi concetti di egemonia e ordine internazionale, ha a che fare con la natura di questa organizzazione, e riguarda l'elemento (terra/acqua) nel quale, e dal quale, l'ordine è dettato. Già a prima vista, non può sfuggire che le potenze indicate come egemoni - Portogallo, Olanda, Gran Bretagna e Stati Uniti - sono tutte potenze navali. Esse possiedono quelli che Modelski (1983) definisce i «fattori dell'egemonia mondiale»: 1) una posizione geografica favorevole, peninsulare o preferibilmente insu-

9 Non si deve dimenticare, anzi, che la Dottrina Monroe venne spesso riconosciuta come «il primo e finora il più riuscito esempio del principio di un grande spazio internazionale» (Schmitt 1939, cit. in Portinaro 1982a, 191), e che la rivendicazione di un analogo spazio per la Germania fu appoggiata proprio al precedente storico del Western Hemisphere. Su questo tema, cfr. Schmitt (1991).

10 Sulla relazione tra espansione commerciale ed espansione navale, cfr. Ratzel (1906), Mahan (1890), Seeley (1971). 
lare; 2) una società politicamente stabile, coesa e aperta; 3) una economia «leader»; 4) una organizzazione politico-strategica in grado di proiettare la propria potenza su scala globale (global reach). È questo insieme di caratteristiche, assenti nelle potenze continentali ${ }^{11}$, ciò che induce la potenza egemone a impegnarsi per il mantenimento dell'ordine internazionale. I quattro fattori si alimentano reciprocamente. L'insularità, come insegna una tradizione ininterrotta di riflessione politica, favorisce la libertà politica (Montesquieu 1973; Seeley 1971; Dehio 1988); l'una e l'altra insieme hanno un legame esistenziale con il commercio e la libertà economica (Hegel 1974; Marx 1978); infine, la capacità di innovazione tecnologica (che è ciò che connota, più delle dimensioni assolute, una economia leader) (Rapkin 1987, 143) consente lo sviluppo degli strumenti politico-strategici per l'azione globale - in concreto: la potenza navale e, oggi, quella aerea e spaziale.

L'opposizione tra ordini e potenze navali e ordini e potenze continentali occupa un posto di riguardo tanto nella dinamica storica, quanto nella riflessione politica e filosofica che l'ha accompagnata $^{12}$. La lotta tra le due condizioni storiche d'esistenza e le relative visioni del mondo è simboleggiata dai conflitti «gemelli» tra Sparta e Atene, Roma e Cartagine, Francia e Inghilterra, Germania e Inghilterra, Unione Sovietica e Stati Uniti, e si rispecchia nel dibattito fra i teorici del «primato del mare» dalla riflessione tucididea sulla «talassocrazia» a quella dell'Am-

11 Sul rapporto tra globalità e navalità, si vedano le osservazioni parallele di due autori per altri versi tanto diversi come Ratzel e Marx. «La ristretta politica territoriale» scrive Ratzel $(1906,39)$ «è, secondo essi, miope; il mare straniero, lontano allarga non solo lo sguardo del commerciante, ma anche quello dell'uomo di Stato. Soltanto il mare può allevare vere forze mondiali. La universale grandezza storica di Roma incomincia dapprima realizzando l'idea che una grande Potenza dev'essere anche Potenza marittima, e le numerose vittorie nello sviluppo di Roma, costituiscono l'unione della terra e del mare, unione che ruppe per sempre i monopoli marittimi dei Cartaginesi e dei Greci. Ad Atene, potente per mare, v'è stata sempre una concezione più grecamente grandiosa che presso la limitata Sparta». Analogamente in Marx (1978, 79): «Per un sistema basato sulle usurpazioni locali, bastava la terra; per un sistema basato sull'aggressione universale, l'acqua diventava indispensabile».

12 Questa polarità orizzontale tra i due elementi può essere, ed è stata spesso, risolta in senso vettoriale (Bolaffi 1986,13$)$. La storia della civiltà si muoverebbe, da una prospettiva di «storia universale» (Weltgeschichte), dalla terra verso il mare e, seguendo lo stesso impulso, da Est verso Ovest: «La storia del mondo» come afferma Hegel nelle Lezioni sulla filosofia della storia $(1941,233$, cit. in Bolaffi 1986, 14) «va da oriente a occidente: l'Europa è infatti assolutamente la fine della storia del mondo così come l'Asia ne è il principio». L'ordine navale costituirebbe, da quest'ottica, la liquidazione, e il superamento, degli ordini continentali. 
miraglio Mahan sulla centralità del sea power - e i teorici del «primato della terra» - fino alla teorizzazione di Halford Mackinder sulla «terra centrale» e sulla possibilità che ha la potenza terrestre di trasformare il mare in un «lago chiuso», impadronendosi di tutte le basi navali (Mackinder 1919, 48-49).

In questo confronto, la posizione delle teorie egemoniche del mutamento non lascia margini al dubbio. Sebbene siano sempre esistiti, ed esistano ancora, ordini continentali, l'egemonia globale è sempre, dal loro angolo di visuale, una egemonia navale. Il potere militare rimane essenziale, ma cambia natura, e valore, a seconda dello spazio. Gli strumenti «tellurici» adatti alla conquista territoriale e al dominio diretto non si prestano altrettanto bene al governo del sistema. Se il potere continentale (land power) può restare preminente a livello regionale, al livello globale il migliore indicatore del potere diventa, per l'intero corso della storia moderna, il potere navale (sea power) (Modelski 1987, 5). La teoria dei lunghi cicli trae, da ciò, una decisiva implicazione empirica. Concentrazione e deconcentrazione della potenza non sono più misurati sulla base dei tradizionali criteri di territorio, popolazione, prodotto interno lordo o generica forza militare, ma sulla base del possesso di navi da guerra. La dimensione della polarità acquista una connotazione più precisa. Ciò che muove il lungo ciclo non è la distribuzione del potere in generale, ma la distribuzione del potere navale ${ }^{13}$. Da un lato, l'ordine internazionale è tanto più saldo quanto più il potere navale è concentrato nelle mani della potenza egemone. Dall'altro lato, e in conseguenza di ciò, la causa fondamentale delle guerre generali è la costruzione di flotte rivali (solo navali in origine, oggi anche aeree e missilistiche), cioè dell'unico strumento in grado di minacciare la posizione di monopolio del più forte.

L'appropriazione del mare (Seenabme) è il sostituto egemonico dell'appropriazione della terra. Non importa che, da una

13 Cercando di dare un fondamento empirico-quantitativo a queste tesi, Thompson ha misurato le variazioni nel possesso delle flotte militari negli ultimi quattro secoli. Da queste ricerche, lo studioso statunitense ha tratto due serie diverse di dati (cit. in Goldstein 1988, 132). La prima si riferisce agli anni nei quali una sola potenza ha posseduto più del $50 \%$ del potere navale mondiale: $1502-1544,1608-1619,1624,1632$ $1633,1635-1636,1640-1642,1719-1723,1809-1812,1814-1834,1843,1854-1857,1861$, 1880-1881, 1889-1890, 1944 a oggi. La seconda, invece, si riferisce agli anni nei quali le nuove potenze egemoni superarono le vecchie nel possesso della flotta: 1602 (Olanda); 1676 (Inghilterra); 1941 (Stati Uniti). 
prospettiva di storia universale, «simili estensioni del diritto nello spazio del mare libero sono avvenimenti (...) di portata rivoluzionaria» (Schmitt 1991, 22). Quello che importa è che, a seguito della Seenabme delle potenze marinare protestanti, olandesi prima, inglesi poi e nordamericani nel nostro secolo hanno potuto promuovere un ordine internazionale diverso, e alternativo, rispetto a quello delle potenze continentali. I suoi tratti distintivi hanno un legame essenziale con la globalità. Il primo, e fondamentale, è l'esclusività. L'ordine navale tende a costituire un «universo» politico, fondato sull'egemonia della potenza marittima e sul divieto, per le altre, di sfidarla sul suo terreno ${ }^{14}$. Come scriveva Ratzel nel suo saggio sul «mare come origine della grandezza dei popoli» (1906, 56-57), «ciascuna Potenza navale decade nel monopolismo. (...) Dacché esiste la storia, una sola Potenza non ha dominato ancora l'intero mondo conosciuto, ma certamente sono esistiti momenti, ne' quali restò un'unica flotta da guerra ed un solo dominio marittimo; ciò avvenne alla distruzione di Cartagine ed alla caduta di Napoleone». Mentre l'appropriazione di terra non esclude, e anzi si trova a convivere, con altre appropriazioni, l'appropriazione del mare, per essere davvero tale, esclude la ripartizione. Per la stessa ragione per la quale «il mare non ha carattere, nel senso originario del termine, che deriva dal greco charassein, scavare, incidere, imprimere» (Schmitt 1991, 20), esso può passare dalla libertà al monopolio, dall'essere di tutti all'essere di uno solo.

L'altra faccia dell'esclusività, e l'ultimo tratto distintivo del concetto di ordine racchiuso nelle teorie egemoniche, è la particolare attitudine nei confronti dell'apertura e della chiusura dello spazio. Pur con diverse accentuazioni, tanto la teoria della stabilità egemonica, quanto la teoria dei lunghi cicli, quanto la

14 La sensibilità delle egemonie marittime nei confronti della costruzione di flotte (potenzialmente) rivali è uno dei più caratteristici fattori di continuità della loro politica estera. Basti pensare alle reazioni che la seconda legge navale tedesca del 1900 provocò in Gran Bretagna, e che spinsero i due paesi a cercare (senza successo), tra il 1908 e il 1909 , un accordo sulle percentuali, per ottenere il quale la Germania avrebbe accettato una proporzione di 3:4 che la Gran Bretagna trovò inaccettabile (Salvatorelli 1975, 156157 e 492-493); agli accordi di Washington del 1922, che sanzionarono i mutati equilibri nel potere navale nella proporzione di 5:5:3 tra Stati Uniti, Gran Bretagna e Giappone, e riservarono a Francia e Italia una quota di 1,75 (Salvatorelli 1975, 732-733); oppure, più recentemente, alle preoccupazioni degli Stati Uniti per ogni estensione della presenza navale sovietica nell'Oceano Pacifico, nel Mar Baltico e, a partire dagli anni settanta, nello stesso Mediterraneo; o a quelle che sta suscitando oggi il rafforzamento e la ristrutturazione della flotta cinese. 
teoria della guerra e del mutamento internazionale di Gilpin presuppongono l'esistenza di un sistema economico internazionale capitalista, anzi ne adottano per così dire l'immagine dello spazio: una cartografia omogenea, unificata in tutte le direzioni da una rete di scambi indifferenti per vocazione a tutti i confini. Il principio della «porta aperta» merita di essere considerato il riassunto simbolico della natura totale dell'egemonia. In esso convergono tutte e tre le sue dimensioni essenziali: la natura tendenzialmente universale del commercio mondiale, e la sua vocazione ad abbattere tutti i limiti politici, culturali e religiosi; il carattere «vicario» che assume, dalla sua ottica, il potere militare, il quale non a caso prende a raffigurarsi come polizia più che come politica internazionale; infine, l'idoneità a trasformare la teoria e la pratica del commercio mondiale in un principio di portata universale, che non è possibile rifiutare senza porsi al di fuori della «comunità internazionale» ${ }^{15}$.

Questa attitudine peculiare nei confronti dello spazio è la vera e propria architrave del nuovo concetto di ordine internazionale. Da un lato, essa spiega la continua oscillazione tra l'accento sull'apertura e quello sulla stabilità (Cesa 1996, 46-47), che la teoria della stabilità egemonica condivide con la maggior parte dell'analisi più recente della politica internazionale. Apertura e stabilità figurano, in realtà, come le due facce della stessa medaglia. «Il punto di vista americano» come ha scritto recentemente uno studioso (Ikenberry 1996), ricostruendo l'origine dell'ordine internazionale post-bellico, «era che l'apertura economica fosse un elemento essenziale per un ordine politico stabile e pacifico».

15 È significativo che questa pretesa appaia sin dal principio dell'espansione marittima europea nel Nuovo Mondo. Posto di fronte al problema di trovare un titolo giuridico per l'occupazione spagnola dell'America, già Francisco de Vitoria si era appellato all'obbligo per gli indigeni di non opporsi al diritto di ospitalità e alla libertà di missione, al liberum commercium e alla libera propaganda (Schmitt 1991). Ma se, nel teologo spagnolo, il liberum commercium non aveva ancora nulla a che fare con il principio liberale del libero commercio mondiale, già cinquant'anni più tardi la sua argomentazione cominciò a piegare in una direzione del tutto nuova. Nella controversia che oppose il portoghese Freitas e l'olandese Grozio (Alexandrowitz 1967) - espressione entrambi, quindi, di due potenze navali - il primo insistette ancora sull'obbligo, per i sovrani delle Indie Orientali, di tenere la porta aperta al diritto dei portoghesi di propagare la fede e la civiltà cristiana. Il secondo, invece, abbandonando del tutto il tradizionale argomento religioso - al quale oppose, anzi, un generale relativismo - pervenne allo stesso risultato in nome del «diritto naturale» al libero commercio. Sia l'uno che l'altro giustificarono la pretesa europea di trovare aperta la porta dell'India: ma mentre in Freitas riecheggia ancora l'idea pre-moderna della guerra al servizio della fede, in Grozio la guerra legittima è già quella - egemonica - contro i disturbatori della pax mercatorum. 
Dall'altro lato, e soprattutto, questo legame tra apertura e stabilità aggiunge un nuovo, e decisivo elemento all'opposizione tra egemonia mondiale e imperi continentali. La prima procede con l'apertura dello spazio, e opera con ogni chiusura (politica, culturale e, in ultima istanza, commerciale) come liquidazione. Il suo elemento, il mare - come il suo strumento, la penetrazione economica - «confina strettamente alle terre che nazionalmente si richiudono in se stesse. Esso si spinge entro esse, separa l'uno dall'altro i territori nazionali e porta il carattere internazionale perfino sopra piccole strisce di terra e sugli istmi» (Ratzel 1906, 41). La sua immagine dello spazio è segnata dalla continuità, dalla «liscia uniformità del mare»: una superficie senza ostacoli, sulla quale la strategia si risolve in geometria (Schmitt 1991, 372).

I secondi, al contrario, si reggono sulla discontinuità tra il proprio spazio e quello esterno, vale a dire, su una delimitazione in senso spaziale (un bortus clausus). Questa attitudine opposta nei riguardi dello spazio si riflette nella natura delle rispettive crisi. La crisi, o la fine, della pace egemonica coincide con l'innalzamento, o la rinascita, delle fratture che l'egemonia aveva rimosso; la fine della pace imperiale, al contrario, procede dalla caduta della frattura che l'impero aveva istituito. In un caso, la pace è spazzata via dal venir meno della continuità. Nell'altro, la pace è travolta dall'abbattimento del limite, e dall'irruzione del consueto nello spazio dal quale era stato escluso.

Dissoluzione della pace egemonica e dissoluzione della pace imperiale si rivelano, in definitiva, fenomeni speculari. Al tramonto della pace egemonica le porte, che dovevano restare aperte, si chiudono. Al tramonto della pace imperiale le porte, che dovevano restare chiuse, si aprono.

\section{Conclusioni}

Le teorie egemoniche dell'ordine internazionale sono state oggetto di numerose critiche, tanto sul versante empirico quanto su quello strettamente teorico. Queste critiche hanno investito aspetti diversi, dal legame tra egemonia e libero commercio alle periodizzazioni storiche, dalla definizione dell'ordine come bene pubblico all'enfasi sulle risorse materiali. Con una certa dose di approssimazione, tuttavia, si può dire che esse si siano concentrate sui presupposti «realisti» delle tre teorie, inseren- 
dosi nel consueto dibattito tra neorealismo e neoliberalismo. Le critiche liberali alle teorie egemoniche offrono una diversa interpretazione del rapporto tra ordine ed egemonia, una diversa interpretazione del mutamento internazionale e, soprattutto, una diversa prognosi del futuro. In primo luogo, esse contestano il fatto che la stabilità e l'apertura del sistema capitalistico mondiale siano dipese, e dipendano, dalla presenza di un paese egemone (gli Stati Uniti). In secondo luogo, e in conseguenza di ciò, esse separano i due mutamenti che le teorie realiste considerano essenzialmente legati: l'ascesa e il declino delle grandi potenze, e la formazione e la crisi dei rispettivi ordini internazionali. Infine, per non dimenticare il sottinteso politico del dibattito, i liberali sono molto più ottimisti dei realisti sulle sorti dell'ordine internazionale post-bellico. «Cinquant'anni dopo la sua nascita, il mondo liberal-democratico occidentale è ancora saldo, e i suoi principi e le sue politiche rimangono il centro dell'ordine globale. Le sfide al multilateralismo liberale sia dall'interno che dall'esterno sono in gran parte scomparse... Le forze dell'integrazione commerciale e finanziaria stanno muovendo inesorabilmente il mondo verso un sistema sempre più strettamente interconnesso indifferente a tutti i confini, sia regionali che nazionali» (Ikenberry 1996).

Queste differenze, per quanto significative, non devono fare perdere di vista il fatto che le critiche liberali hanno in comune con le teorie realiste proprio la cosa più importante: il concetto di ordine internazionale. Sebbene mettano in dubbio la sua dipendenza dall'egemonia, infatti, anche per i liberali l'ordine internazionale è inconcepibile se non come un fatto globale, occidentale, e fondato sul principio dell'apertura economica. Il superamento delle divisioni economiche, in origine, ma poi anche culturali e politiche, assume anzi i tratti di una specie di filosofia della storia, la quale trova alimento nella fiducia nella modernizzazione diffusasi a partire dagli anni cinquanta nelle scienze sociali, ma con forti addentellati nelle filosofie della storia ottocentesche; nell'utopia liberale e borghese della pace attraverso il commercio; in quella «idealista», che di quest'ultima costituisce il complemento istituzionale, delle organizzazioni internazionali di tipo universale; nell'idea stessa della trasferibilità del modello «occidentale» di mercato e democrazia.

Come in molti altri settori delle Relazioni Internazionali (Wendt 1992), pur non concordando su buona parte delle spiegazioni, neorealisti e neoliberali hanno in comune gli stessi pre- 
supposti e, soprattutto, la stessa prospettiva etnocentrica. «Naturalmente» come ha scritto recentemente un critico severo (Smith 1995, 23) «non è indifferente che gli stati cerchino guadagni relativi o assoluti, così come è importante sia praticamente che teoricamente il ruolo delle istituzioni internazionali. Ma pretendere che in un mondo di pericoli e violenze estreme queste siano le questioni cruciali per la teoria internazionale appare perverso... Il dibattito è un dibattito occidentale, anzi atlantico. Esso sfiora appena le preoccupazioni della maggior parte dell'umanità, e passa sotto silenzio tutto ciò che non trova posto in questa visione americana di ciò che conta nella politica internazionale».

Le conseguenze di questa «restrizione dell'orizzonte» investono tutti gli aspetti più significativi dell'analisi del mutamento. In primo luogo, l'accento sulla globalità e sulla navalità induce le teorie egemoniche a ignorare fenomeni macroscopici, solo perché avvengono al di fuori, o «al di sotto», della lotta per l'egemonia globale. Basti pensare al silenzio della teoria dei lunghi cicli sulla guerra costituente per eccellenza del sistema moderno di relazioni internazionali, la guerra dei Trent'anni, che finisce eclissata all'interno del ciclo egemonico olandese (Levy 1990) - e proprio nella fase di massimo ordine! ${ }^{16}$ Sul mare, dove si svolge il gioco dell'egemonia, non c'è eco di ciò che avviene nel continente, anche quando questo cambia alla radice la teoria e la prassi della convivenza internazionale.

In secondo luogo, questa visione unitaria, e miope, dell'ordine internazionale, rende imprendibile l'ultimo grande mutamento del Novecento: il collasso dell'Unione Sovietica e del sistema internazionale bipolare. E sufficiente gettare uno sguardo sulle teorie egemoniche per rendersi conto di come la loro idea dell'ordine e dell'egemonia globale prescindesse già, sin dall'inizio, dall'Unione Sovietica. Stabilità e apertura dell'ordine internazionale del dopo-guerra erano riferiti esplicitamente all'Occidente liberale e capitalista, rispetto al quale l'Unione Sovietica e il suo «impero informale» (Wendt e Friedheim 1995; Lake 1996) costituivano tutt'al più uno sfidante, un limite o un mondo dietro al mondo.

Non può stupire che, da questo angolo di visuale, la fine

16 Seguendo una periodizzazione non molto lontana da quella della teoria dei lunghi cicli, Wallerstein colloca l'egemonia olandese tra il 1625 e il 1675. 
dell'Unione Sovietica appaia meno come la fine di un ordine, quello bipolare, che come la rimozione dell'unico intralcio all'ordine liberale degli anni quaranta. La sottovalutazione, se non la rimozione, della cesura dell'89 può anzi essere considerata una delle tendenze più pervasive dell'analisi recente delle Relazioni Internazionali, oltre che una delle più chiare dimostrazioni della sua impronta «atlantica». In un articolo apparso pochi mesi fa su «Foreign Affairs» questa tesi compare nella sua forma «chimicamente pura»:

Per l'opinione comune, la fine della guerra fredda ha costituito uno spartiacque storico, perché il collasso del comunismo ha trascinato con sé quello dell'ordine che aveva preso forma dopo la Seconda Guerra mondiale... L'opinione comune si sbaglia. Ciò che è finito con la guerra fredda è solo il bipolarismo... Ma l'ordine mondiale creato a metà degli anni quaranta perdura, più ampio e per certi versi persino più saldo che durante gli anni della guerra fredda (Ikenberry 1996).

Ma quello che conta di più è che, escludendo dal proprio orizzonte tutto ciò che non rimette in discussione l'ordine globale, le teorie del mutamento condannano all'invisibilità aree sempre più consistenti della realtà internazionale, dai processi di frammentazione a quelli di riaggregazione regionale, dai fenomeni di mobilitazione etnica e micro-nazionale ai processi di region-building, dai problemi del sottosviluppo al collasso di singoli stati o intere regioni. Una volta che la fine dell'Unione Sovietica ha chiuso la lotta («egemonica») per il governo del sistema, il mutamento internazionale non scompare dalla realtà, ma finisce segregato in quella zona grigia della politica internazionale che i liberali liquidano come anacronistica, e i neorealisti come non pertinente.

\section{Riferimenti bibliografici}

Alexandrowitz, C.H. (1967), An Introduction to the History of the Law of Nations in the East Indies (16th, 17th and 18th centuries), Oxford, Clarendon Press.

Aron, R. (1970), Pace e guerra tra le nazioni, Milano, Comunità.

Baldwin, D.O. (1986), Analisi del potere e politica internazionale, in Bonanate e Santoro (1986), pp. 205-242.

Baumgartner, T., W. Buckley e T.R. Burns (1975), Meta-power and Relational Control in Social Life, in «Social Science Information», 14, pp. $49-78$. 
Belligni, S. (1983), Voce «Egemonia», in AA.VV., Dizionario di politica, Torino, Utet, pp. 371-373.

Bolaffi, A. (1986), Introduzione, in Schmitt (1986).

Bonanate, L. (1986), Sistema politico internazionale, in Bonanate e Santoro (1986), pp. 119-130.

Bonanate, L. e C.M. Santoro (a cura di) (1986), Teoria e analisi nelle relazioni internazionali, Bologna, Il Mulino.

Braudel, F. (1966), Il mondo attuale, Torino, Einaudi.

Carr, E.H. (1951), The Twenty Years Crisis, 1919-1939. An Introduction to the Study of International Relations, London, Macmillan.

Cesa, M. (1990), L'equilibrio internazionale: Modelli a confronto, in «Il Politico», n. 1, pp. 229-249.

- (1996), Politica e economia internazionale, Milano, Jaca Book.

Chase-Dunn, C. (1990), World-state Formation: Historical Processes and Emergent Necessity, in «Political Geography Quarterly», vol. 9 , n. 2, pp. 108-130.

Christensen, C. (1977), Structural Power and National Security, in K. Knorr e F.N. Trager (a cura di), Economic Issues and National Security, Lawrence, Kansas, University Press.

Dahl, R.A. (1970), Modern Political Analysis, Englewood Cliffs, Prentice-Hall.

Dehio, L. (1988), Equilibrio o egemonia. Considerazioni sopra un problema fondamentale della storia politica moderna, Bologna, Il Mulino.

Doyle, M. (1986), Empires, Ithaca, Cornell, University Press.

Freund, J. (1986), L'essence du politique, Paris, Sirey.

Galtung, J. (1966), East-West Interactions Patterns, in «Journal of Peace Research», n. 2, pp. 146-176.

Gilpin, R. (1989), Guerra e mutamento nella politica internazionale, Bologna, Il Mulino.

Goldstein, J.S. (1988), Long Cycles. Prosperity and War in the Modern Age, New Haven-London, Yale, University Press.

Grabowski, A. (1960), Raum, Staat und Geschichte. Grundlegung der Geopolitik, Colonia, Heymann.

Hegel, G.W.F. (1941), Lezioni sulla filosofia della storia, Firenze, La Nuova Italia.

Herz, J. (1951), Political Realism and Political Idealism, Chicago, Chicago, University Press.

Hirsch, F. e M.W. Doyle (1977), Politicization in the World Economy; Necessary Conditions for an International Economic Order, in F. Hirsch e M.W. Doyle, Alternatives to Monetary Disorder, New York, McGraw-Hill.

Holsti, K.J. (1971), Retreat from Utopia: International Relations Theory, 1945-70, in «Canadial Journal of Political Sciences», n. 4, pp. 165-177. 
Houweling, H.W. e J.G. Siccama (1993), A Neo-functionalist Theory of War, in «International Interactions», vol. 18, pp. 387-408.

Ikenberry, G.J. (1996), The Myth of the Post-Cold War Chaos, in «Foreign Affairs», vol. 75, n. 3, pp. 79-90.

Ikenberry, G.J. e C.A. Kupchan (1990), The Legitimation of Hegemonic Power, in D.P. Rapkin (a cura di), World Leadership and Hegemony, London, Rienner, pp. 49-70.

Jervis, R. (1978), Cooperation under the Security Dilemma, in «World Politics», XXX, pp. 167-214.

Kaplan, M. (1957), System and Process in International Politics, Reading, Addison-Wesley.

Kennedy, P. (1989), Ascesa e declino delle grandi potenze, Milano, Garzanti.

Keohane, R.O. (1980), The Theory of Hegemonic Stability and Changes in International Economic Regimes, 1967-1977, in O.R. Holsti, R.M. Siverson e A.L. George (a cura di), Changes in the International System, Boulder, Westview Press.

- (1984), After Hegemony: Cooperation and Discord in the World Political Economy, Princeton, Princeton, University Press.

Keohane, R.O. e J.S. Nye (1977), Power and Interdependence: World Politics in Transition, Boston, Little Brown.

Kindleberger, C.P. (1973), The World in Depression, 1929-1939, Berkeley, California University Press.

- (1981), Dominance and Leadership in the International Economy, in «International Studies Quarterly», 25, pp. 242-254.

Krasner, S.D. (1976), State Power and the Structure of International Trade, in «World Politics», 28, pp. 317-347.

- (1986), Cause strutturali e conseguenze del concetto di regime, in Bonanate e Santoro (1986), pp. 131-154.

Kumon, S. (1987), The Theory of Long Cycles Examined, in Modelski (1987), pp. 56-84.

Lake, D.A. (1996), Anarchy, Hierarchy, and the Variety of International Relations, in «International Organization», vol. 50, n. 1, pp. 1-33.

Lasswell, H. e A. Kaplan (1950), Power and Society, New Haven, Yale University Press.

Levy, J.S. (1983), War in the Modern Great Power System, 1495-1975, Lexington.

- (1986), Teorie della guerra generale, in Bonanate e Santoro (1986), pp. 391-424.

- (1990), Long Cycles, Hegemonic Transitions, and the Long Peace, prepared for delivery at the Annual Meeting of the International Studies Association in Washington, D.C.

Mackinder, H.J. (1919), Democratic Ideals and Reality. A Study in the Politics of Reconstruction, London, Constable \& Company.

Mahan, A.T. (1890), The Influence of Sea-Power in History, 16601783, Boston, Little Brown. 
Marx, K. (1978), Storia diplomatica segreta del $18^{\circ}$ secolo, Milano, La Pietra.

Miglio, G. (1988), La sovranità limitata, in G. Miglio, Le regolarità della politica, Milano, Giuffrè, pp. 1009-1074.

Miller, B. (1992), A «New World Order»: From Balancing to Hegemony, Concert or Collective Security?, in «International Interactions», vol. 18, n. 1, pp. 1-33.

Modelski, G. (1978), Long Cycles of Global Politics and the Nation State, in «Comparative Studies in Society and History», vol. 20, n. 2, pp. 214-235.

- (1983), Long Cycles of World Leadership, in W.R.Thompson (a cura di), Contending Approaches to World System Analysis, Beverly Hills, Sage Publications.

- (a cura di) (1987), Exploring Long Cycles, Boulder, Lynne Rienner Publishers.

Montesquieu, C. de (1973), Lo spirito delle leggi, Torino, Utet.

Organski, A.F.K. (1965), The Stages of Political Development, New York, Alfred Knopf.

- (1968), World Politics, New York, Alfred Knopf.

Organski, A.F.K. e J. Kugler (1980), The War Ledger, Chicago, Chicago University Press.

Portinaro, P.P. (1982a), La crisi dello jus publicum europaeum. Saggio su Carl Schmitt, Milano, Comunità.

- (1982b), Nel tramonto dell'Occidente: la geopolitica, in «Comunità», n. 184, ottobre, pp. 1-42.

Rapkin, D.P. (1987), World Leadership, in Modelski (1987), pp. 129-157.

Ratzel, F. (1906), Il mare origine della grandezza tra i popoli, Torino, Unione Tipografico-Editrice.

Russett, B. (1985), The Mysterious Case of Vanishing Hegemony; or, Is Mark Twain Really Dead?, in «International Organization», vol. 39, n. 2, pp. 207-231.

Russett, B. e H. Starr (1992), La politica mondiale. Introduzione allo studio delle relazioni internazionali, Bologna, Il Mulino.

Salvatorelli, L. (1975), Storia del Novecento, Milano, Mondadori.

Santoro, C.M. (1988), Il sistema di guerra. Studi sul bipolarismo, Milano, Angeli.

- (1992), Introduzione, in Russett e Starr (1992).

- (1995), I nuovi poli geopolitici, in «Relazioni Internazionali», $\mathrm{n}$. 33, pp. 4-17.

Schmitt, C. (1939), Völkerrechtliche Grossraumordnung mit Interventionsverbot für Raumfremde Mächte. Ein Beitrag zum Reichsbegriff im Völkerrecht, Berlin, Deutscher Rechtsverlag.

Schmitt, C. (1986), Terra e mare, Milano, Giuffrè.

- (1991), Il Nomos della terra, Milano, Giuffrè.

Schumpeter, J.A. (1979), Storia dell'analisi economica, Torino, Boringhieri. 
Seeley, J.R. (1971), The Expansion of England, Chicago-London, Chicago, University Press.

Singer, J.D. (1980), Accounting for International War: The State of the Discipline, in «Journal of Peace Research», vol. 18, n. 1, pp. 1-18.

Siverson, R. e M.P. Sullivan (1983), The Distribution of Power and the Onset of War, in «Journal of Conflict Resolution», vol. 27, n. 3, pp. 473-494.

Smith, S. (1995), The Self-Images of a Discipline: A Genealogy of International Relations Theory, in K. Booth e S. Smith (a cura di), International Relations Theory Today, University Park, Pennsylvania, Pennsylvania State, University Press.

Sorokin, P. (1937), Social and Cultural Dynamics, vol. 3, Fluctuations of Social Relationships, War and Revolution, New York, American Book Company.

Thompson, W.R. e G. Modelski (1994), Long Cycle Critiques and Deja Vu All Over Again: A Rejoinder to Houweling and Siccama, in «International Interactions», vol. 20, n. 3, pp. 209-222.

Toynbee, A. (1934), A Study of History, London.

Tucidide (1989), La guerra del Peloponneso, Milano, Rizzoli.

Tucker, R.C. (1981), Politics as Leadership, Columbia, Missouri University Press.

Wallerstein, I. (1982), Il sistema mondiale dell'economia moderna, Bologna, Il Mulino.

Waltz, K. (1987), Teoria della politica internazionale, Bologna, Il Mulino.

Wendt, A. (1992), Anarchy is What States Make of It: The Social Construction of Power Politics, in «International Organization», vol. 46, n. 2, pp. 391-425.

Wendt, A. e D. Friedheim (1995), Hierarchy under Anarchy: Informal Empire and the East German State, in «International Organization», vol. 49, n. 4, pp. 689-721.

Wright, Q. (1965), A Study of War, Chicago, Chicago, University Press.

Young, O.R. (1991), Political Leadership and Regime Formation: on the Development of Institutions in International Society, in «International Organization», vol. 45, n. 3, pp. 281-318.

Zorzi, R. (1982), Tramonto dell'Occidente e geopolitica, in «Comunità», n. 184, pp. V-IL. 